\title{
Clonal strains of Pseudomonas aeruginosa in paediatric and adult cystic fibrosis units
}

\author{
M.R. O'Carroll*, M.W. Syrmis ${ }^{\#}$, C.E. Wainwright ${ }^{\oplus}$, R.M. Greer ${ }^{+}$, P. Mitchell ${ }^{\oplus}$, C. Coulter ${ }^{\S}$, T.P. Sloots ${ }^{\#}$, \\ M.D. Nissen ${ }^{\#, \S}$, S.C. Bell*,f
}

Clonal strains of Pseudomonas aeruginosa in paediatric and adult cystic fibrosis units. M.R. O'Carroll, M.W. Syrmis, C.E. Wainwright, R.M. Greer, P. Mitchell, C. Coulter, T.P. Sloots, M.D. Nissen, S.C. Bell. (C)ERS Journals Ltd 2004.

ABSTRACT: Despite recent reports of clonal strains of Pseudomonas aeruginosa in cystic fibrosis (CF) units, the need for routine microbiological surveillance remains contentious.

Sputum was collected prospectively from productive patients attending the regional paediatric and adult CF units in Brisbane, Australia. All $P$. aeruginosa isolates were typed using pulsed-field gel electrophoresis. Spirometry, anthropometrics, hospitalisations and antibiotic sensitivity data were recorded.

The first 100 sputum samples (first 50 patients at each clinic) harboured 163 isolates of $P$. aeruginosa. A total of 39 patients shared a common strain (pulsotype 2), 20 patients shared a strain with at least one other patient and 41 patients harboured unique strains. Eight patients shared a strain identical to a previously reported Australian transmissible strain (pulsotype 1). Compared with the unique strain group, patients harbouring pulsotype 2 were younger and had poorer lung function. Treatment requirements were similar in these two groups, as were the rates of multiresistance.

In conclusion, $59 \%$ of patients harboured a clonal strain, supporting the need for routine microbiological surveillance. In contrast to previously described clonal strains, the dominant pulsotype was indistinguishable from nonclonal strains with respect to both colonial morphology and multiresistance. The clinical significance of clonal strains remains uncertain and requires longitudinal study.

Eur Respir J 2004; 24: 101-106.
*Adult Cystic Fibrosis Unit, The Prince Charles Hospital, ${ }^{\#}$ Clinical Virology Research Unit, Sir Albert Sakzewski Virology Research Centre, TDept of Respiratory Medicine, Royal Children's Hospital, ${ }^{+}$Paediatric and Child Health, ${ }^{f}$ Dept of Medicine, University of Queensland and ${ }^{\$}$ Queensland Health Pathology Service, Queensland Health, Brisbane, Australia.

Correspondence: S.C. Bell, Thoracic Physician, Dept of Thoracic Medicine, The Prince Charles Hospital, Rode Road, Chermside 4032, Australia. Fax: 61732125630

E-mail: scott_bell@health.qld.gov.au

Keywords: Clonal strain, cystic fibrosis, Pseudomonas aeruginosa, pulsed-field gel electrophoresis

Received: November 22003

Accepted after revision: February 132004

This study was supported by the Royal Children's Hospital Foundation Seeding grant R912-007, which was sponsored by the Cressbrook Committee.
Advances over the past three decades have significantly improved survival for patients with cystic fibrosis (CF). Nonetheless, chronic pulmonary infection with Pseudomonas aeruginosa has remained the most important cause of both morbidity and mortality in $\mathrm{CF}$ [1]. $P$. aeruginosa is a ubiquitous organism that is capable of adapting to the unique environment of the CF lung. It is able to assume a mucoid phenotype and create a biofilm, which enhances bacterial resistance to phagocytosis and antipseudomonal antibiotics. Once established in the CF lung, $P$. aeruginosa is virtually impossible to eradicate. Although the age of $P$. aeruginosa acquisition is an important determinant of prognosis [2], transmission from one patient to another is historically thought to be an uncommon occurrence [3, 4] and, accordingly, segregation based on infection status is not routine.

Numerous studies have demonstrated that people with $\mathrm{CF}$ typically harbour their own unique strain of $P$. aeruginosa, which is presumably acquired from the environment during childhood $[5,6]$. Subsequent acquisition of other strains and the sharing of strains are considered unusual, with the exception of siblings where this occurrence is well-documented [7-9]. Several recent reports [10-13], including two from Australia, have described clonal strains of $P$. aeruginosa in patients attending CF centres. With the exception of an early Danish study [14], which lacked reliable bacterial fingerprinting, the first such clonal strain was only confirmed by molecular typing methods, after a progressive increase in the prevalence of ceftazidime-resistant $P$. aeruginosa led to further investigation [12]. Subsequently, interest in the possible existence of clonal strains has grown, leading to the discovery of several other clonal strains within CF-care settings. In each instance, a single dominant strain has prevailed. The clonal strain has typically, but not invariably, been of mucoid phenotype and associated with increased antibiotic resistance. The mode of transmission has presumably been patient-topatient spread, as earlier reports have not identified an environmental reservoir $[12,13]$. Recently, two studies have reported evidence that clonal strains may be more virulent and potentially portend a worse prognosis $[11,15]$.

The accepted definitive molecular typing method for $P$. aeruginosa is restriction fragment length polymorphism analysis by pulsed-field gel electrophoresis (PFGE). Not all groups employing PFGE have been able to demonstrate the presence of a clonal strain of $P$. aeruginosa within their $\mathrm{CF}$ clinics [16], leading to debate about the clinical significance of these strains [17, 18]. The current authors aimed to determine if clonal strains were prevalent in the regional paediatric and adult CF centres for Queensland, Australia, and whether the same strains occurred in both clinical settings.

\section{Methods and materials}

\section{Clinical data}

The cystic fibrosis clinics. The Royal Children's Hospital (RCH) CF clinic manages 270 patients throughout Queensland 
(184 sputum producers, $123(66.8 \%)$ of whom are infected with $P$. aeruginosa). Of the sputum producers, 132 were capable of reproducible pulmonary function testing. Within this group, 78 children had evidence of chronic $P$. aeruginosa infection [19]. Most infants with $\mathrm{CF}$ identified by newborn screening receive care at $\mathrm{RCH}$ and transition to the Prince Charles Hospital (PCH) between the ages of 16-18 yrs. Inpatients without chronic $P$. aeruginosa infection were, at the time of this study, not segregated from those with $P$. aeruginosa and routinely shared rooms. Dining facilities and exercise equipment were also shared. Patients infected with Burkholderia cepacia complex or methicillin-resistant Staphylococcus aureus (MRSA) are nursed in single rooms as inpatients, and are also segregated in the outpatient clinic and use separate lung function equipment. No facilities are shared with the adult clinic.

The PCH CF clinic manages 151 patients from throughout Queensland; 129 have evidence of chronic P. aeruginosa infection [19]. Patients are admitted for infective exacerbations and typically receive 10-14 days of parenteral antibiotic therapy. Where circumstances permit, it is routine practice to manage patients in single rooms. Infection control measures for MRSA and B. cepacia complex are otherwise the same as for the $\mathrm{RCH}$.

Patients, samples and data collection. Specimens were collected between December 2001 and July 2002, and consisted of spontaneously produced sputum collected either at outpatient review or during hospital admission. Isolates of $P$. aeruginosa were obtained from the sputum samples of the first 50 patients collected at each centre (100 of 283 patients in total).

Clinical data were obtained by medical record review. All measurements were taken from the point closest to the date of sputum collection. The clinical characteristics of the 100 patients were compared with the respective study populations to determine whether they were representative samples. In addition, comparisons were made between patients with chronic $P$. aeruginosa infection at both sites. The definition of chronic $P$. aeruginosa infection was that reported by LEE et al. [19]. Further clinical data were then collected from the study cohort of 100 patients. This included CF transmembrane regulating protein genotype, days of inpatient care and i.v. antibiotic administration in the preceding 12 months, use of recombinant DNAase (rhDNAase), routine use of inhaled antibiotics and all lung function measurements for the preceding 2 yrs. The best forced expiratory volume in one second (FEV1) \% predicted in the preceding 24 months was recorded and the rate of decline in FEV1 \% pred $\left(\% \cdot \mathrm{yr}^{-1}\right)$ was modelled using linear regression. Rate of decline in FEV1\% pred was omitted from the analysis for three patients, due to either extreme rates of change or insufficient data points (three or fewer).

Approval for this prospective study was obtained from the ethics committees of both the $\mathrm{RCH}$ and $\mathrm{PCH}$. Written informed consent was obtained from all $\mathrm{CF}$ patients aged $\geqslant 12$ yrs with $P$. aeruginosa infection and from the parents of all children aged $<18$ yrs.

\section{Microbiology}

Sputum processing and antimicrobial sensitivity testing. Sputum samples were processed routinely in the respective centres. Sputum was cultured on selective and nonselective media, and isolates were identified as $P$. aeruginosa based on compatible morphology and a positive oxidase reaction, and growth at $42^{\circ}$ C. Selective-culture media routinely used include: sheep blood agar (bioMerieux Australia, Baulkam Hills, Australia); chocolate bacitracin agar (containing $\mathrm{BBL}^{\mathrm{TM}}$ blood agar base infusion agar (BD Australia/New Zealand, North Ryde, Australia), defibrinated horse blood (bioMerieux Australia) and bacitracin zinc salt (Sigma-Aldrich, Castle Hill, Australia)); Maconkey agar with Crystal Violet (bioMerieux Australia); B. cepacia complex medium (Mast Diagnostics, Merseyside, UK); and $\mathrm{BBL}^{\mathrm{TM}}$ mannitol salt agar (BD Australia/New Zealand). Suspect colonies, which failed to grow at $42^{\circ} \mathrm{C}$, were identified using the API 20NE (bioMérieux Vitek, Hazelwood, MO, USA) identification system. Identification was confirmed with an oprL PCR, using methods previously described $[20,21]$. A single colony representative of each morphological type was subcultured onto sheep blood agar (Accumedia, Baltimore, MD, USA) for further study.

Antibiotic susceptibility testing was performed by discdiffusion testing on $\mathrm{BBL}^{\mathrm{TM}}$ Mueller Hinton II agar (BD Australia/New Zealand) according to the National Committee for Clinical Laboratory Standards [22]. Colistinsusceptibility testing was performed using a disc containing $10-\mu \mathrm{g}$ colistin sulphate and interpreted according to previously proposed criteria [23]. All antibiotic sensitivity testing was conducted at the PCH for purposes of standardisation. The criteria for multiresistance were those proposed by a Cystic Fibrosis Foundation Consensus Conference; namely, a $P$. aeruginosa isolate from a patient with $\mathrm{CF}$ is defined as multiresistant if it is resistant to all agents in two of the following classes of antibiotics: the $\beta$-lactams, the aminoglycosides, and/or the quinolones [24]. Co-pathogens were recorded.

Pulsed-field gel electrophoresis. Stored isolates from the 100 patients were subject to molecular typing by PFGE, performed by a scientist blinded to clinical status, using the methods previously described by SPENCKER et al. [4]. Plugs were digested with the restriction enzyme SpeI and electrophoresis was performed with a clamped homogenous electric fieldsDRIII module (Bio-Rad Laboratories, Hercules, CA, USA) with modification of the switch times. Similarity analysis of results was calculated. Cluster analyses of the similarity matrices were generated using the unweighted pair group method using arithmetic averages. Criterion for related clones for all assays was taken as profiles with $\geqslant 90 \%$ similar bands. Banding patterns were also compared visually and the Tenover criteria [25] were employed to describe relatedness. The greatest number of band differences between isolates categorised as "related" was three. As an Australian clonal strain has previously been reported as pulsotype $1[10,11]$, the current authors' subsequent strains were labelled from pulsotype 2. For the purpose of analysis, if pulsotype 2 was present with any other genotype, the patient was classified into pulsotype 2 group. Patients classified into the unique genotype group had no evidence of infection with another clonal strain or cluster (including pulsotype 1 or 2 ). In the absence of definitive epidemiological evidence of the transmission of $P$. aeruginosa from patient to patient or the environment to patient, the term "clonal strain" has been used in this study and based on molecular definitions detailed previously. Minor clonal strains occurring in two or more patients were termed as a cluster.

\section{Statistics}

The samples of 50 patients taken from each clinic were compared with their respective populations using unpaired $\mathrm{t}$-tests with a significance level of 0.05 , except for the number 
of admissions, which were compared using Wilcoxon's rank sum test. The clinical characteristics of the two groups harbouring unique and dominant strains were compared, using analysis of covariance to adjust for the effects of age and clinic. Adult and paediatric patients were grouped together in the analysis. Wilcoxon's rank sum test was used to compare the total number of inpatient days and i.v. antibiotic days, as these were not normally distributed. Chisquared and Fisher's exact test (if there were fewer than five observations) were employed to compare differences in the antimicrobial-resistance rates. Results are presented as adjusted means (95\% confidence interval) for normally distributed variables or as median (interquartile range) for number of hospital admissions, inpatient days and days on i.v. antibiotics, unless otherwise specified.

\section{Results}

\section{Clinical data}

The mean $\pm \mathrm{SD}$ age of the paediatric patients was $12.0 \pm$ $3.1 \mathrm{yrs}$ and $48 \%$ were female, as compared to $11.3 \pm 3.5 \mathrm{yrs}$ and $52.3 \%$ female for the whole clinic. This paediatric sample was subject to more frequent hospitalisation in the previous 12 months $(1.0(0-3.0)$ versus $0.0(0-1.5) ; \mathrm{p}=0.0002)$ when compared to sputum-producing children who were able to perform lung function tests within the clinic $(n=132)$. When compared to children who had chronic $P$. aeruginosa infection $(\mathrm{n}=78)$, there was no difference in age, FEV1\% pred, forced vital capacity (FVC) \% pred, weight z-score, height z-score and number of admissions in the previous year (data not shown).

The mean \pm SD age of the adults was $28.5 \pm 9.1 \mathrm{yrs}$ and $42.0 \%$ were female, as compared with $26.1 \pm 7.9$ yrs and $41.7 \%$ female for the whole clinic. The patients studied had lower mean FEV1 \% pred $(48.2 \pm 19.6 \%$ versus $55.5 \pm 23.6$; $\mathrm{p}=0.0497)$ and more frequent hospital admissions $(2.0$ (0-3.0) versus 1.0 $(0-3.0) ; \mathrm{p}=0.04)$ than the clinic group as a whole $(\mathrm{n}=151)$. When compared with adults who had chronic $P$. aeruginosa infection $(n=129)$, there were no significant differences in age, FEV1 \% pred, FVC \% pred, weight, height and number of admissions in the previous year (data not shown).

The majority of patients $(82 \%)$ had their sputum collected as outpatients. Out of the 18 patients that were inpatients, 10 harboured pulsotype 2 , five had unique strains and three had minor clusters.

\section{Microbiology}

Strains of P. aeruginosa. A total of 163 isolates of $P$. aeruginosa were cultured from the sputum of 100 patients (1-3 isolates per patient). The results of the visual comparison of PFGE profiles were concordant. Each of the banding patterns was compared with all other banding patterns. In total, 58 unique banding patterns were identified. A dominant strain (pulsotype 2) was identified in sputum from 39 patients (63 isolates). Out of the 63 isolates, 46 were identical and the remainder had evidence of between one and three band differences by PFGE. Four patients harboured pulsotype 2, as well as a further strain: pulsotype 58 in one patient, two patients had unique strains and one patient had two unique strains. A further 41 patients harboured unique strains (65 isolates) and the remaining 20 patients shared five clusters (30 isolates) with at least one other person. Eight patients (five adults, three children) shared a strain which was indistinguishable from a representative isolate of the previously reported Australian clonal strain (pulsotype 1). Three of the clusters (pulsotypes 3,42 and 58) were contained completely within either the paediatric or the adult clinic, and involved between two and seven patients. The final strain (pulsotype 5) was shared between one patient from each clinic. There was no close contact between any of the patients who shared the minor clusters. In the case of pulsotype 5, the patients never had any contact, including having never attended the same hospital. Out of the 20 patients sharing minor clusters, one had co-infection with a unique $P$. aeruginosa strain (pulsotype 3 and unique strain) and one harboured two of these minor strains (pulsotype 1 and 58).

Out of 39 patients, $25(64.1 \%)$ harbouring pulsotype 2 attended the paediatric clinic, as compared with $34.2 \%$ of those with unique strains $\left(\chi^{2}=7.42, \mathrm{p}=0.02\right)$. Of the 14 adults with pulsotype 2 , eight $(57.1 \%)$ received their paediatric care at the $\mathrm{RCH}$, as compared with $29.6 \%$ of those harbouring a unique strain $\left(\chi^{2}=3.0, \mathrm{p}=0.24\right)$. Of the six adults with pulsotype 2 who had not attended the RCH for care, four had admissions to the adult clinic that overlapped with patients known to have pulsotype-2 infections.

When compared with those harbouring a unique strain, the pulsotype-2 group had a significantly lower FEV1\% pred and FVC $\%$ pred at the time of sputum collection (table 1). The best FEV1 \% pred recorded in the previous 24 months, the rate of decline in lung function, anthropometric measures and treatment requirements were similar for the two groups. There were no significant differences in any of these measures when the group harbouring pulsotype 1 was compared with the unique strain group.

The potential confounding effects of infection with $B$. cepacia complex or MRSA were examined by repeating the analysis of clinical outcome variables with these patients excluded. The results did not change when those harbouring B. cepacia complex were omitted; however, when the patients harbouring MRSA were excluded, the pulsotype-2 group had significantly more inpatient days in comparison with the unique strain group ( $\mathrm{p}=0.03$ ). When all four patients harbouring these co-pathogens were omitted, the pulsotype-2 group

Table 1.-Clinical characteristics of the pulsotype groups

Pulsotype 2 Unique strains p-value

\begin{tabular}{|c|c|c|c|}
\hline Total patients $n$ & 39 & 41 & \\
\hline Adults/children $n$ & $14 / 25$ & $27 / 14$ & 0.02 \\
\hline Age yrs & $16.8 \pm 7.3$ & $24.6 \pm 12.9$ & 0.002 \\
\hline Female $\%$ & 43.6 & 51.2 & $0.52^{\#}$ \\
\hline$\Delta \mathrm{F} 508$ homozygotes $\%$ & 48.7 & 41.5 & $0.25^{\#}$ \\
\hline Inhaled $\mathrm{Ab} \%$ & 66.7 & 56.1 & $0.33^{\#}$ \\
\hline Inhaled RhDNAase \% & 53.8 & 43.9 & $0.37^{\#}$ \\
\hline FEV1 \% pred $^{+}$ & $54.2(48.0-60.5)$ & $63.8(57.6-70.0)$ & 0.04 \\
\hline Best FEV1 $\%$ pred $^{+}$ & $69.8(63.9-75.7)$ & $73.7(68.0-79.5)$ & 0.34 \\
\hline FVC $\%$ pred $^{+}$ & $65.2(59.5-70.8)$ & $75.3(69.8-80.9)$ & 0.02 \\
\hline FEV 1 decline $\% \cdot \mathrm{yr}^{-1+}$ & $-3.2(-4.9--1.6)$ & $-1.9(-3.6--0.3)$ & 0.28 \\
\hline Total n & 39 & 38 & \\
\hline Weight $\mathrm{z}$-score & $-0.61 \pm 0.92$ & $-0.28 \pm 1.02$ & 0.3 \\
\hline Height z-score & $-0.57 \pm 0.86$ & $-0.14 \pm 1.12$ & 0.3 \\
\hline Median inpatient days ${ }^{\S}$ & $26.0(9.0-54.0)$ & $14.0(0-38.0)$ & 0.07 \\
\hline Median i.v. Ab days & $26.0(14.0-66.0)$ & $15.0(0-56.0)$ & 0.27 \\
\hline
\end{tabular}

Data are presented as $\mathrm{n}$, mean $(95 \% \mathrm{CI})$ adjusted for age and clinic, mean $\pm \mathrm{SD}$, and median (interquartile range) unless otherwise stated. Comparisons were made using analysis of covariance unless otherwise stated. The Kruskal-Wallis test was used to compare inpatient days and i.v. antibiotic $(\mathrm{Ab})$ days in the preceding 12 months. RhDNAase: recombinant DNAase; FEV1: forced expiratory volume in one second; FVC: forced vital capacity. \#: comparison using Chi-squared test; ": outlying values (three patients were excluded from the analysis due to insufficient data points and extreme rates of change); ${ }^{+}$: mean $(95 \% \mathrm{CI})$; §. median (interquartile range). 
Table 2. - Antimicrobial resistance according to pulsotype

\begin{tabular}{lccc}
\hline Antibiotic & Pulsotype 2 & Unique strains & p-value \\
\hline Total n & 63 & 65 & \\
Gentamicin & 93.7 & 61.5 & $<0.0001$ \\
Tobramycin & 58.7 & 29.1 & 0.0008 \\
Amikacin & 87.3 & 53.8 & $<0.0001$ \\
Ceftazidime & 42.9 & 26.2 & 0.05 \\
Ticarcillin/clavulanic acid & 47.6 & 24.6 & 0.006 \\
Piperacillin & 54.0 & 27.7 & 0.002 \\
Ciprofloxacin & 39.7 & 27.7 & 0.15 \\
Aztreonam & 31.8 & 40.0 & 0.33 \\
Meropenem & 34.9 & 9.2 & 0.0004 \\
Imipenem & 33.3 & 16.9 & 0.03 \\
Colistin & 4.8 & 0 & $0.10^{\#}$ \\
Multiresistant & 28.6 & 16.9 & 0.12 \\
\hline
\end{tabular}

Data are presented as $\mathrm{n}$ and $\%$ of isolates resistant to the given antibiotic unless otherwise stated. Chi-squared tests were used to compare resistance rates unless otherwise stated. \# : Fisher's exact test was used to compare resistance rates.

had significantly more inpatient days, as compared with those in the unique strain group $(26.5(9.0-54.0)$ versus 12.0 $(0.0-36.0) ; \mathrm{p}=0.02)$.

Phenotype and antibiotic resistance. In total, $44 \%$ of all isolates were of mucoid phenotype. The results of antibioticsensitivity testing are shown in table 2. Pulsotype-2 $P$. aeruginosa was more resistant to all aminoglycosides, ceftazidime, ticarcillin/clavulanic acid, piperacillin, meropenem and imipenem, when compared to the unique strains. The rates of multiresistance were similar in the pulsotype-2 and unique strain groups. Three of the pulsotype-2 isolates (from two children) were resistant to colistin. This was confirmed with E-test minimum inhibitory bactericidal concentrations. Both of these patients had routinely received inhaled colistin, but never the i.v. form. Considering mucoidy in the pulsotype- 2 and unique strain groups, 33 of $63(52.4 \%)$ of pulsotype $2 \mathrm{had}$ mucoid phenotype, as compared with 25 of $65(38.5 \%)$ of the unique strain $\left(\chi^{2}=2.5, \mathrm{p}=0.11\right)$.

Co-pathogens. A total of 42 patients had another pathogen detected in sputum (S. aureus $(\mathrm{n}=29)$, MRSA $(\mathrm{n}=4)$, Aspergillus fumigatus $(\mathrm{n}=9), B$. cepacia complex $(\mathrm{n}=2)$ and Achromobacter xylosoxidans $(\mathrm{n}=1)$ ). Two adult patients harbouring pulsotype 2 had co-infection with B. cepacia complex and MRSA, respectively. Although the patient infected with B. cepacia complex had always been segregated at the adult clinic, she had received her paediatric care at the $\mathrm{RCH}$ and had not been segregated prior to the identification of B. cepacia complex. The patient with MRSA had always been segregated at the adult clinic and had never attended the paediatric clinic.

\section{Discussion}

Experience from $\mathrm{CF}$ clinics is highly variable with respect to the potential for patient-to-patient spread of $P$. aeruginosa $[11,16,18]$, leading to widely disparate views about the prevalence and significance of clonal strains. Whilst three groups have provided convincing evidence of clonal strains of $P$. aeruginosa within their clinics [11-13], others have not, despite utilisation of the same molecular typing techniques [16]. The current authors have found evidence of a dominant clonal strain of $P$. aeruginosa shared across the regional paediatric and adult CF centres in Queensland, Australia, in this prospective cross-sectional study. In total, $59 \%$ of the patients harboured a clonal strain. Whilst a paediatric clonal strain has been previously described within an adult CF clinic [26], to the current authors' knowledge, this is the first study to attempt to investigate the extent of $P$. aeruginosa transmission across geographically distinct clinics. The current findings provide further evidence that clonal strains of $P$. aeruginosa are an important issue and it is believed that this problem will continue to emerge and pose significant clinical and infection-control dilemmas for many, but not necessarily all, $\mathrm{CF}$ units.

Increased antibiotic resistance (including multiresistance) and an unusual phenotype (different colonial morphology) have characterised the clonal strains of $P$. aeruginosa reported to date [11-13]. The current authors' pulsotype 2 had a phenotype indistinguishable from unique strains, but was more likely to be resistant to various antibiotics, albeit not multiresistant. A mucoid phenotype did not emerge as a hallmark. The only unusual phenotypic feature was the presence of colistin resistance in three isolates from two paediatric patients. Both of these patients had routinely used inhaled colistin, but never received the i.v. form. This may, therefore, represent resistance induced by antibiotic selection. Accurate estimates of the prevalence of colistin resistance amongst $P$. aeruginosa isolates are limited, although a recent paper by PITT et al. [27] described colistin resistance in 3\% of $P$. aeruginosa isolates obtained from 17 hospitals throughout the UK. Another recently published study from the UK found higher levels of baseline colistin resistance (25-32\%) [28] and further highlighted the difficulty of using conventional breakpoints to define resistance in relation to colistin and other inhaled antibiotics [29]. DENTON et al. [30] reported the transmission of colistin-resistant $P$. aeruginosa amongst paediatric CF patients and genotyping confirmed four of the isolates to be indistinguishable by PFGE. In all other respects, the presence of the pulsotype-2 strain within the clinics would probably have been overlooked if molecular investigation had not been performed.

Six adult patients harbouring pulsotype 2 had never attended the paediatric clinic. Four of these had hospital admissions to the PCH that overlapped with patients known to be infected with pulsotype 2. One of the six patients had chronic infection with MRSA and had always been segregated at the $\mathrm{PCH}$, in addition to never having attended the paediatric clinic. The presence of pulsotype 2 in the adult clinic, therefore, cannot be solely explained by the transfer of infected paediatric patients, suggesting that transmission of the clonal strain may have occurred independently within both clinics. Environmental acquisition remains possible and could explain the identification of pulsotype 2 in the patient infected with MRSA. The Manchester group have recently isolated epidemic $P$. aeruginosa from room air when patients performed spirometry, nebulisation and airway clearance, suggesting that aerosol dissemination may be the most important factor in patient-to-patient spread [31]. It may prove that both person-to-person transmission and acquisition from an environmental source have occurred concurrently, but this requires prospective study. An alternative explanation for the possible transmission of pulsotype 2 in the adult clinic is patient contact in the outpatient clinic or other hospital areas outside the ward.

SPEERT et al. [16] recently reported multiple clusters of CF patients who shared identical strains of $P$. aeruginosa. These clusters consisted of between two and 21 patients and epidemiological evidence to support patient-to-patient spread could be found in only one pair of unrelated individuals. The current authors also found several clusters shared amongst unrelated individuals. The majority of these patients have had no direct social contact outside the hospital setting. Whether these shared strains represent the result of patient-to-patient 
spread or acquisition from a common environmental source remains contentious. One of these clusters consisted of eight patients harbouring pulsotype 1, the Australian transmissible strain first described in Melbourne and more recently in Sydney. Four of these patients had previously lived and received medical care in Sydney, and a fifth attended school with a CF child attending the Sydney clinic. Pulsotype 2 has recently been identified in patients attending $\mathrm{CF}$ clinics in Sydney (B. Rose, Dept of Infectious Diseases, University of Sydney, New South Wales, Australia, person communication). Thus, there is evidence that these strains are not confined to isolated centres. This highlights the importance of appropriate communication of microbiological status when patients move from one clinic to another.

The clinical implications of clonal strains of $P$. aeruginosa remain unclear. It seems likely that there is considerable geographical variability and that these strains are not a universal phenomenon. The findings that patients harbouring the Manchester clonal strain have increased treatment requirements [15] and that the pulsotype-1 strain was associated with more severe disease in young patients [11] provide preliminary evidence that some of these strains may have an adverse impact on clinical status. However, it seems probable that the degree of virulence of individual clonal strains will be strain specific. The current authors' pulsotype 2 was associated with poorer lung function, which may equally reflect an inpatient bias, as much as any potential harmful effects, and it requires prospective study. As a result of these findings, infection control policies at the current authors' two units have been modified. The units conform to Australian Cystic Fibrosis Centre Infection Control guidelines. Strict segregation of patients with $P$. aeruginosa and those with no evidence of $P$. aeruginosa infection in outpatient clinics and during inpatient treatment is now practiced. It remains feasible that person-to-person spread (including during social contact between patients) of the clonal strain of $P$. aeruginosa may occur despite this policy. Specific segregation based on $P$. aeruginosa genotype is not currently performed, which is consistent with published infection control recommendations for patients with $\mathrm{CF}$ [32]. The adoption of such measures is likely to have significant resource implications given current facilities and the ongoing cost of $P$. aeruginosa genotype screening. Whilst it is far from certain that all clonal strains are detrimental, the potential for transmission of such strains, regardless of pathogenicity, at the very least mandates the separation of this patient group from those without $P$. aeruginosa infection, which in turn is best achieved by employing the infection control recommendations described previously.

This study provides further support for longitudinal molecular surveillance of Pseudomonas aeruginosa and ongoing monitoring to screen for super-infection within cystic fibrosis clinics. This parallels recommendations of the Cystic Fibrosis Trust (UK) Control of Infection Group for such surveillance [33]. The widespread application of longitudinal screening programmes will enhance understanding of the significance of clonal strains of Pseudomonas aeruginosa.

Acknowledgements. The authors would like to thank J. Faoagali and the staff of the microbiology laboratories at the Prince Charles Hospital and the Royal Brisbane Hospital. The authors would also like to thank T. Kidd for performing the antimicrobial sensitivity testing and the physiotherapists and specialist nurses in the respective cystic fibrosis units for their assistance in specimen collection. The authors thank J. Govan, K. Webb, A. Jones and B. Rose for providing epidemic strains of Pseudomonas aeruginosa.

\section{References}

1. Govan JR, Deretic V. Microbial pathogenesis in cystic fibrosis: mucoid Pseudomonas aeruginosa and Burkholderia cepacia. Microbiol Rev 1996; 60: 539-574.

2. Nixon GM, Armstrong DS, Carzino R, et al. Clinical outcome after early Pseudomonas aeruginosa infection in cystic fibrosis. J Pediatr 2001; 38: 699-704.

3. Govan JR. Infection control in cystic fibrosis: methicillinresistant Staphylococcus aureus, Pseudomonas aeruginosa and the Burkholderia cepacia complex. J R Soc Med 2000; 93: Suppl. 38, 40-45.

4. Spencker FB, Haupt S, Claros MC, et al. Epidemiologic characterization of Pseudomonas aeruginosa in patients with cystic fibrosis. Clin Microbiol Infect 2000; 6: 600-607.

5. Romling U, Fiedler B, Bobhammer J, et al. Epidemiology of chronic Pseudomonas aeruginosa infections in cystic fibrosis. J Infect Dis 1994; 170: 1616-1621.

6. Mahenthiralingam E, Campbell ME, Foster J, Lam JS, Speert DP. Random amplified polymorphic DNA typing of Pseudomonas aeruginosa isolates recovered from patients with cystic fibrosis. J Clin Microbiol 1996; 34: 1129-1135.

7. Grothues D, Koopmann U, von der Hardt H, Tummler B. Genome fingerprinting of Pseudomonas aeruginosa indicates colonization of cystic fibrosis siblings with closely related strains. J Clin Microbiol 1988; 26: 1973-1977.

8. Tummler B, Koopmann U, Grothues D, Weissbrodt H, Steinkamp G, von der Hardt H. Nosocomial acquisition of Pseudomonas aeruginosa by cystic fibrosis patients. $J$ Clin Microbiol 1991; 29: 1265-1267.

9. Wolz C, Kiosz G, Ogle JW, et al. Pseudomonas aeruginosa cross-colonization and persistence in patients with cystic fibrosis: use of a DNA probe. Epidemiol Infect 1989; 102: 205-214.

10. Anthony M, Rose B, Pegler MB, et al. Genetic analysis of Pseudomonas aeruginosa isolates from the sputa of Australian adult cystic fibrosis patients. $J$ Clin Microbiol 2002; 40: 2772-2778.

11. Armstrong DS, Nixon GM, Carzino R, et al. Detection of a widespread clone of Pseudomonas aeruginosa in a pediatric cystic fibrosis clinic. Am J Respir Crit Care Med 2002; 166: 983-987.

12. Cheng K, Smyth RL, Govan JR, et al. Spread of betalactam-resistant Pseudomonas aeruginosa in a cystic fibrosis clinic. Lancet 1996; 348: 639-642.

13. Jones AM, Govan JR, Doherty CJ, et al. Spread of a multiresistant strain of Pseudomonas aeruginosa in an adult cystic fibrosis clinic. Lancet 2001; 358: 557-558.

14. Pedersen SS, Koch C, Hoiby N, Rosendal K. An epidemic spread of multiresistant Pseudomonas aeruginosa in a cystic fibrosis centre. J Antimicrob Chemother 1986; 17: 505-516.

15. Jones AM, Dodd ME, Doherty CJ, Govan JR, Webb AK. Increased treatment requirements of patients with cystic fibrosis who harbour a highly transmissible strain of Pseudomonas aeruginosa. Thorax 2002; 57: 924-925.

16. Speert DP, Campbell ME, Henry DA, et al. Epidemiology of Pseudomonas aeruginosa in cystic fibrosis in British Columbia, Canada. Am J Respir Crit Care Med 2002; 166: 988-993.

17. Pitt TL. Cross infection of cystic fibrosis patients with Pseudomonas aeruginosa. Thorax 2002; 57: 921.

18. Ramsey BW. To cohort or not to cohort: how transmissible is Pseudomonas aeruginosa? Am J Respir Crit Care Med 2002; 166: 906-907.

19. Lee TWR, Brownlee KG, Conway SP, Denton M, Littlewood JM. Evaluation of a new definition for chronic Pseudomonas aeruginosa infection in cystic fibrosis patients. J Cystic Fibrosis 2003; 2: 29-34.

20. De Vos D, Lim A Jr, Pirnay JP, et al. Direct detection and identification of Pseudomonas aeruginosa in clinical samples such as skin biopsy specimens and expectorations by multiplex PCR based on two outer membrane lipoprotein genes, oprI and oprL. J Clin Microbiol 1997; 35: 1295-1299. 
21. Pirnay JP, De Vos D, Duinslaeger L, et al. Quantitation of Pseudomonas aeruginosa in wound biopsy samples: from bacterial culture to rapid real-time polymerase chain reaction. Crit Care 2000; 4: 255-261.

22. The National Committee for Clinical Laboratory Standards. Twelfth International Supplement M100-S12 Vol. 22 No. 1: Performance standards for antimicrobial susceptibility testing. Wayne, PA, USA, 2002.

23. Gales AC, Reis AO, Jones RN. Contemporary assessment of antimicrobial susceptibility testing methods for polymyxin $\mathrm{B}$ and colistin: review of available interpretative criteria and quality control guidelines. J Clin Microbiol 2001; 39: $183-190$

24. Cystic fibrosis consensus conference. Microbiology and infectious disease in cystic fibrosis. Bethesda, MD, Cystic Fibrosis Foundation, 1994; Vol. 5, Section 1, pp. 1-26.

25. Tenover FC, Arbeit RD, Goering RV. How to select and interpret molecular strain typing methods for epidemiological studies of bacterial infections: a review for healthcare epidemiologists. Molecular typing working group of the Society for Healthcare Epidemiology of America. Infect Control Hosp Epidemiol 1997; 18: 426-439.

26. McCallum SJ, Corkill J, Gallagher M, Ledson MJ, Hart CA, Walshaw MJ. Superinfection with a transmissible strain of Pseudomonas aeruginosa in adults with cystic fibrosis chronically infected by $P$. aeruginosa. Lancet 2001; 358: 558-560.
27. Pitt TL, Sparrow M, Warner M, Stefanidou M. Survey of resistance of Pseudomonas aeruginosa from UK patients with cystic fibrosis to six commonly prescribed antimicrobial agents. Thorax 2003; 58: 794-796.

28. Hodson ME, Gallagher CG, Govan JRW. A randomised clinical trial of nebulised tobramycin or colistin in cystic fibrosis. Eur Resp J 2002; 20: 658-664.

29. Govan JRW. Insights into cystic fibrosis microbiology from the European tobramycin trial in cystic fibrosis. J Cystic Fibrosis 2002; 1: Suppl. 2, S203-S208.

30. Denton M, Kerr K, Mooney L, et al. Transmission of colistin-resistant Pseudomonas aeruginosa between patients attending a paediatric cystic fibrosis center. Pediatr Pulmonol 2002; 34: 257-261.

31. Jones AM, Govan JR, Doherty CJ, et al. Identification of airborne dissemination of epidemic multi-resistant strains of Pseudomonas aeruginosa at a CF centre during a crossinfection outbreak. Thorax 2003; 58: 525-527.

32. Saiman L, Siegel J, for the Cystic Fibrosis Foundation. Infection control recommendations for patients with cystic fibrosis: microbiology, important pathogens, and infection control practises to prevent patient-to-patient transmission. Infect Control Hosp Epidemiol 2003; 24: Suppl. 5, S1-S52.

33. The CF Trust's Control of Infection Group. Pseudomonas aeruginosa infection in people with cystic fibrosis. Kent, UK, Cystic Fibrosis Trust, 2001; pp. 1-18. 